\title{
Ein neues Diagnosekonzept für die Praxis
}

\section{Das Multiaxiale Diagnosesystem Jugendhilfe (MAD-J)}

André Jacob, Karl Wahlen und Heike Morche

Das Multiaxiale Diagnosesystem Jugendhilfe (MAD-J) soll es Praktikern der Sozialen Arbeit erleichtern, das Erziehungsverhalten von Eltern zu beurteilen und gegebenenfalls geeignete Hilfen zu finden.

Familien können in schwierigen, die elterliche Erziehung beeinträchtigenden Lebenssituationen zur Stärkung der Erziehungskompetenz der Eltern und zur Unterstützung des Entwicklungsprozesses ihrer Kinder sozialpädagogische, psychologische, beraterische und/oder (psycho) therapeutische »Hilfen zur Erziehung « in Anspruch nehmen. Doch dabei stellen sich zahlreiche Fragen:

- Welche Hilfe ist für wen und unter welchen Umständen geeignet?

- Wie lassen sich die Erziehungsbedingungen, das Erziehungsverhalten und die Entwicklungsprozesse in Familien erfassen?

- Wie findet man die angemessene Intervention?

- Woran erkennt man, dass die Hilfeleistung jeweils »notwendig und geeignet « ist oder war?

Auf diese Fragen müssen in den Hilfeplanungsprozessen der Kinder- und Jugendhilfe Antworten gefunden werden in jedem Einzelfall, aber auch in grundsätzlicher Hinsicht. »Hilfen zur Hilfeplanung « bietet das »Multiaxiale Diagnosesystem Jugendhilfe MAD-J« (2006). Es besteht im Kern aus einem Klassifikationssystem elterlicher Erziehung und ermöglicht die differenzierte Beschreibung und Bewertung der Erziehungs- und Entwicklungssituation eines Kindes oder Ju-

Dr. André Jacob ist Leiter der Erziehungsberatungsstelle des Deutschen Roten Kreuzes in BerlinSteglitz-Zehlendorf. Heike Morche ist dort Mitarbeiterin. Karl Wablen ist Leiter der Psychosozialen Dienste des Jugendamtes Berlin-Neukölln. E-Mail jacoba@drk-berlin.net gendlichen, der Erziehungsbedingungen und der Lebenslage seiner Familie. Aus der entsprechenden, gleichermaßen (entwicklungs-)psychologisch und systemisch begründeten, Diagnose lassen sich Indikationsentscheidungen für Erziehungshilfen mit fachlichen (jugendhilfespezifischen) Begründungen ableiten.

\section{Gegenstand einer jugendhilfe- spezifischen Diagnostik: elterliche Erziehung in Eltern- Kind-Systemen}

Zunächst wollen wir kurz den Zweck und den Gegenstand von Diagnosen in der Jugendhilfe betrachten. Hilfen zur Erziehung sind Mittel, um Störungen in Eltern-Kind-Systemen zu beheben, Eltern in ihrer Erziehungsaufgabe zu unterstützen und die Rechte des Kindes zu schützen (vgl. \1 SGB VIII).

Bei der Planung und Durchführung von Hilfen zur Erziehung werden Diagnosen benötigt, die dem Zweck solcher Hilfen angemessen sind. Im Hilfeplanungsprozess sollten den jugendhilfespezifischen Indikationen deshalb diagnostische Aussagen zugrunde gelegt werden, die sowohl die elterlichen Erziehungsprozesse hinsichtlich ihres Gelingens oder Misslingens beurteilen als auch das Ausmaß und die Qualität der Befriedigung basaler kindlicher Bedürfnisse beschreiben. Darüber hinaus sollten die wichtigsten Erziehungsbedingungen erfasst und in ihren Zusammenhängen beschrieben werden. Dabei lassen sich günstige Bedingungen, die gelingende Erziehung wahrscheinlicher machen und so das Kind in seiner Entwicklung unterstützen, unterscheiden von weniger günstigen, die die Wahrscheinlichkeit von dysfunktionen Erziehungspraktiken und von Entwicklungsbeeinträchtigungen erhöhen.

Die bedingungsanalytischen Modelle von Belsky (1984) und Perrez (2004) und die ihnen zugrunde liegenden Befunde empirischer Untersuchungen machen deutlich: Will man gelingendes oder misslingendes elterliches Erziehungsverhalten beschreiben und operationalisieren, muss eine Fülle von Faktoren berücksichtigt und in komplexen Wirkungszusammenhängen gedacht werden. Ein zum Gegenstandsbereich der Jugendhilfe passendes diagnostisches System benötigt demzufolge eine mehrdimensionale Struktur. Sie muss erstens das elterliche Erziehungsverhalten, zweitens die kindlichen Bedürfnisse und drittens die Bedingungen, unter denen Erziehung stattfindet, abbilden und miteinander in Beziehung setzen.

\section{Der Ansatz: kindliche Bedürfnisse, Erziehung und deren Bedingungen}

Ausgehend von Kellers KomponentenModell elterlichen Erziehungsverhaltens (Keller 2001, 2008) haben Jacob und Wahlen (2006) ein Vier-KomponentenModell der Erziehung in Eltern-Kind-Systemen entwickelt. Darin werden »elterliche Verhaltenssysteme «, »elterliche Interaktionsmechanismen" und "elterliche Affektmuster « als wesentliche Faktoren (Komponenten) des Erziehungshandelns von Eltern unterschieden und in Beziehung gesetzt zu den kindlichen Grundbedürfnissen nach Erhaltung der Vitalität, nach Sicherheit, nach Exploration, nach Kommunikation und nach Lust und Unlustvermeidung. Als vierte Komponente berücksichtigt das Modell die Erfahrungen, die das Kind mit der elterlichen Erziehung macht.

Kulturelle Kontexte liefern die Rahmenstruktur für die Zielorientierung elterlicher Erziehungshandlungen. Wie mit kindlichen Bedürfnissen umgegangen wird, wie sie interpretiert, welche Erziehungshandlungen als adäquate Antwort beurteilt werden und woraufhin eigentlich erzogen werden soll, ist wesentlich durch die Kultur bestimmt, an der die Fa- 
milie teilnimmt. Die Komponentenmodelle von Keller $(2001,2008)$ und von Jacob \& Wahlen (2006) lassen die kulturelle Bedingtheit erzieherischen Handelns nicht außer Acht. Sie haben jedoch den Vorteil, eine Rahmenstruktur zu explizieren, die kulturunabhängig funktioniert und die je nach gesellschaftlichen Normen verschiedene inhaltliche Prägungen und Akzentuierungen annimmt.

Mit Hilfe dieses Ansatzes ist es möglich, Erziehungshandlungen hinsichtlich ihrer wesentlichen Aspekte in eine theoretisch begründete und praxisorientierte Ordnung zu bringen. Fasst man Merkmale elterlicher Erziehungshandlungen in Checklisten zusammen, kann das die Beurteilung des Problemgehalts erzieherischer Verhältnisse und die Bearbeitung entsprechender Fragestellungen unterstützen. In diesem Sinne steht mit dem Multiaxialen Diagnosesystem Jugendhilfe ein diagnostisches Inventar zur Verfügung, das dem Gegenstandsbereich der Jugendhilfe wie den Zwecken und Formen ihrer Hilfeleistung entspricht.

\section{Das Multiaxiale Diagnosesystem Jugendhilfe (MAD-J)}

Das Multiaxiale Diagnosesystem Jugendhilfe ist in fünf Achsen gegliedert. Die Axialität hat den Vorteil, der Komplexität des zu erfassenden Realitätsbereiches eher gerecht zu werden als univariate Verfahren, wie beispielsweise die ICD-10. Das System kann weiterentwickelt werden, ohne seine Grundstruktur verändern zu müssen.
Die Achse I erfasst die »Klinischpsychologische Individualdiagnose « und dient der Beschreibung psychopathologischer kindlicher Merkmale, die oftmals den Beratungsanlass ergeben: beispielsweise Verhaltensauffälligkeiten oder Entwicklungsverzögerungen. Im Unterschied dazu werden auf der Achse III »Medizinische Individualdiagnose des Kindes « Diagnosen notiert, die von Fachleuten des Gesundheitswesens, insbesondere von Ärzten, gestellt wurden und einen deutlichen Zusammenhang zum Vorstellungsgrund in der Erziehungsberatung oder zur Inanspruchnahme anderer Jugendhilfeleistungen aufweisen. Gemeint sind Krankheitsbilder nach ICD-10, DSM-IV oder des Multiaxialen Klassifikationsschemas (MAS) (Remschmidt et al. 2001). Als Beispiele wären hier Störungen des Sozialverhaltens, Essstörungen, Teilleistungsstörungen, Behinderungen und chronische Erkrankungen zu nennen.

Den Kern des Multiaxialen Diagnosesystem Jugendhilfe bildet die Achse II: »Erziehung und deren Bedingungen «. Mit ihrer Hilfe wird der Realitätsbereich abgebildet, auf den sich die gegebenenfalls indizierten Hilfen zur Erziehung beziehen. Die Feststellung von Qualität und Quantität der auf dieser Achse erfassten Merkmale entscheidet, ob Hilfen zur Erziehung indiziert sind und welche Hilfeform für die konkrete Situation geeignet erscheint. Die relevanten Merkmale und ihre Ausprägungen werden auf fünf Subachsen abgebildet:

1. Komponenten der elterlichen Erziehung und kindliche Erziehungserfahrungen: Diese Subachse umfasst die genannten
Verhaltenssysteme, Interaktionsmechanismen und Affektmuster auf der Elternseite sowie die Erfahrungen, die das Kind mit den Erziehungshandlungen seiner Eltern hinsichtlich der beiden Entwicklungs-Dimensionen "Autonomie « und »Bezogenheit « macht.

2. Qualität der Eltern-Kind-Beziehung: Merkmale, die in dieser Hinsicht als wichtig erachtet werden, sind: Akzeptanz, Echtheit, Verständnis, Zuverlässigkeit positiver Kommunikation, Regulationsniveau, elterliche Vorstellung vom Kind, Affektmuster, Rollenverständnis der Eltern als Erziehende und elterliche Zukunftsvorstellungen.

3. Qualität der elterlichen Paarbeziehung: Die Qualität der elterlichen Paarbeziehung als Einflussfaktor auf die Erziehungsfähigkeit wird über die Merkmale Akzeptanz, Echtheit, Verständnis, Stabilität positiver Kommunikationsmuster, Präsenz, Reflexivität, Selbstkontrolle, Rollendiskrimination, Fähigkeit zur Konsensbildung in der Elternrolle, Affektmuster und partnerschaftliche Zufriedenheit ermittelt.

4. Familiäre Organisation: Nach dem »Familienmodell « von Steinhauer und Cierpka (Cierpka/Frevert 1995) werden auf dieser Subachse relevante Eigenschaften des Familiensystems erfasst, insbesondere Aufgabenerfüllung, Rollenverhalten, Kommunikation, Emotionalität, affektive Beziehungsaufnahme, Kontrolle, Werte und Normen in und durch die Familie und deren Subsysteme.

5. Elterliche Personenmerkmale: Relevant sind hier nur Personenmerkmale, die das Erziehungsverhalten beeinflussen. Die

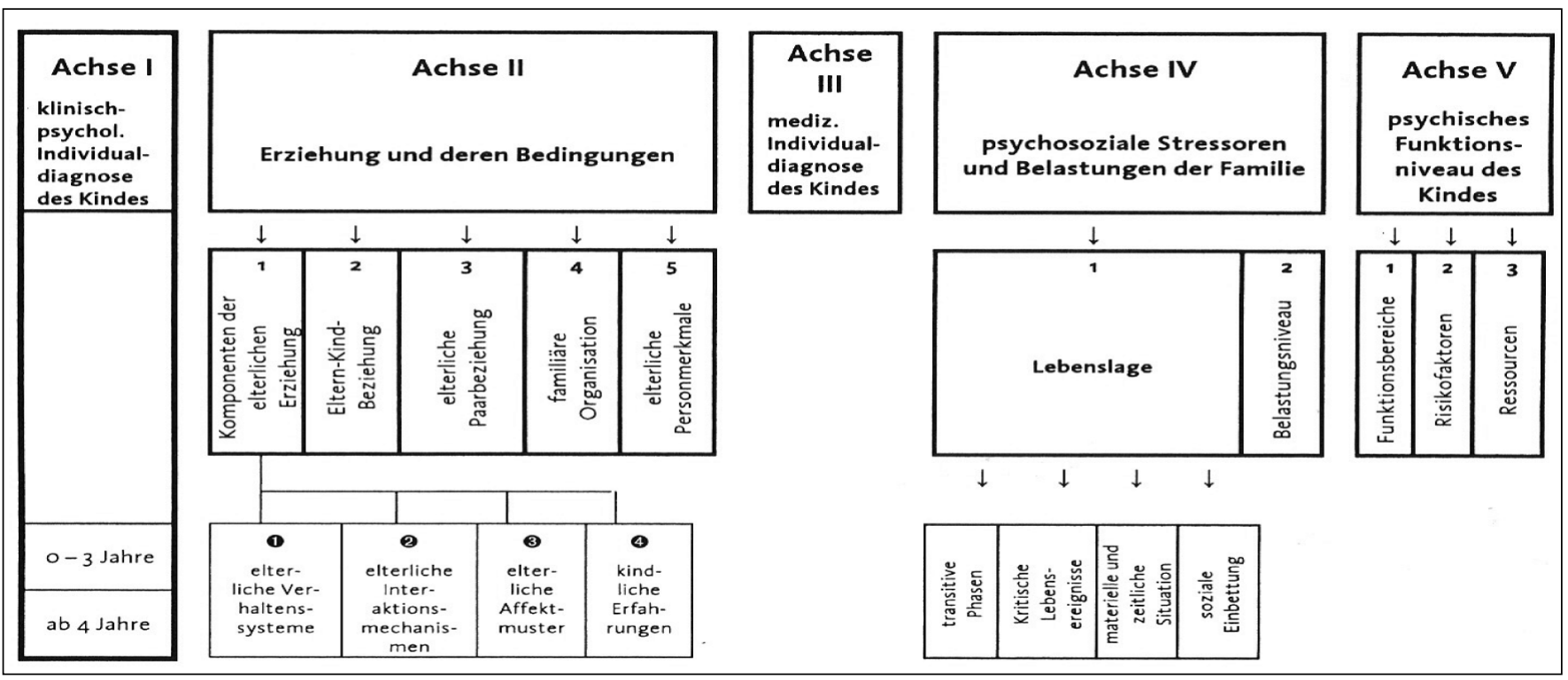


Diagnostik beschränkt sich auf Bereiche persönlicher Abwehr- und Bewältigungsmuster, die Eltern im Umgang mit ihrem Kind beeinflussen können: emotionale Stabilität, Extraversion, soziale Verträglichkeit, Offenheit für Erfahrungen, Gewissenhaftigkeit, Kontrollüberzeugung und Selbstwertschätzung sowie Hilfesuchverhalten.

Über die Achse IV »Psychosoziale Stressoren und Belastungen « und die Achse V "Psychisches Funktionsniveau des Kindes « werden weitere Faktoren aus Belskys (1984) und Perrez (2001) Modell berücksichtigt. Um die familiale Belastungssituation einzuschätzen, werden auf der Achse IV Stressoren aus biografischen Übergängen, aus der materiellen und ökologischen Situation und aus dysfunktionalen sozialen Unterstützungssystemen erfasst. Dane- ben ist die Einschätzung eines globalen Belastungsmaßes möglich.

Die Bewertung des psychischen Funktionsniveaus des Kindes wird auf der Achse $\mathrm{V}$ über verschiedene Funktionsbereiche vorgenommen; es werden Merkmale der Individualität, der Persönlichkeit, der Risikofaktoren und der Ressourcen des Kindes berücksichtigt. Die Abbildung zeigt die Achsenstruktur im Überblick (Seite 234). Eine ausführliche Darstellung geben Jacob und Wahlen (2006).

Die Autoren empfehlen, die Ausprägungen der im MAD-J erfassten Merkmale mit Hilfe einer vierstufigen Ordinalskala A bis $\mathrm{D}$ zu bewerten, die in Anlehnung an die »Parental-Infant-Relation-Global-Assessment Scala « (PIR-GAS, vgl. Zero-ToThree 1998) definiert ist. Dabei bedeutet A: »adaptiv-reguliert/funktional «, B: »un-

\section{Literatur}

Arbeitskreis OPD-KJ (Hg.) (2003): Operationalisierte Psychodynamische Diagnostik im Kindes- und Jugendalter. Verlag Hans Huber, Bern, Göttingen, Toronto, Seattle.

Belsky, J. (1984): The determinants of parenting: A process model. Child development 55, 83-96.

Cierpka, M. und Frevert, G. (1995): Die Familienbögen. Ein Inventar zur Einschätzung von Familienfunktionen. Hogrefe, Göttingen/Bern/Toronto/Seattle.

Fuhrer, U. (2005): Lehrbuch Erziehungspsychologie. Verlag Hans Huber, Bern/Göttingen/Toronto/Seattle.

Jacob, A. und Wahlen, K. (2006): Das multiaxiale Diagnosesystem Jugendhilfe (MAD-J). Personenzentrierte Beratung \& Therapie, 5. Ernst Reinhardt Verlag; München und Basel.

Keller, H. (2001): Frühkindliche Sozialisationskontexte. In: von Schlippe, A. Lösche, G., Hawellek, C. (Hg.) (2001): Frühkindliche Lebenswelten und Erziehungsberatung. Die Chancen des Anfangs. Beltz Verlag, Weinheim, Basel, Berlin, 17-38.

Keller, H. und Chasiotis, A. (2008 im Druck): Entwicklung im Spannungsfeld zwischen Natur und Kultur. In: N. Birbaumer, D. Frey, J. Kuhl, M. Hasselhorn, R. K. Silbereisen (Hg.): Psychologie des Säuglings- und Kindesalters, Enzyklopädie der Psychologie, Band CV4, Hogrefe, Göttingen/Bern/Toronto/Seattle.

Perrez, M. (2004): Stressoren in der Familie und Familie als Stressor im Vorfeld der Entwicklung von Störungen bei Kindern und Jugendlichen. In: Schlottke, P. F., Lauth, G., Sibereisen, R. K., Scheider, S. (Hg.): Enzyklopädie der Psychologie. Band: Störungen im Kindes- und Jugendalter. Hogrefe, Göttingen/Bern/Toronto/Seattle, 193-246.

Remschmidt, H., Schmidt, M. \& Poustka, F. (Hrsg.) (2001): Multiaxiales Klassifikationsschema. 4. Aufl. Verlag Hans Huber, Bern, Göttingen, Toronto, Seattle.

Schneewind, K. A. (1999): Familienpsychologie. 2. Aufl. Verlag W. Kohlhammer, Stuttgart.

Zero To Three National Center for Infants, Toddlers, and Families (1999): Diagnostische Klassifikation: 0-3. Springer, Wien, New York.

Zero To Three National Center for Infants, Toddlers, and Families (2005): Diagnostic Classification of Mental Health and Developmental Disorders of Infacy and Early Childhood; Revised (DC:0-3R). balanciert «, C: »beeinträchtigt« und D: »gestört «. Ein Merkmal wie beispielsweise »Die Eltern strukturieren den Alltag ihres Kindes « soll demnach nicht dichotom beurteilt werden, also nicht bloß im Hinblick darauf, ob es in einem gegebenen Fall vorliegt oder nicht. Vielmehr soll eingeschätzt werden, wie die Eltern den Alltag ihres Kindes strukturieren: funktional (A) oder mehr oder weniger dysfunktional-unausgewogen (B), beeinträchtigt/beeinträchtigend $(\mathrm{C})$ oder gestört (D). Dabei sind Gesichtspunkte der Quantität (im gegebenen Beispiel: zur viel oder zu wenig Struktur), der Qualität (altersangemessene Tagesstrukturierung) und der Performanz (kontinuierliche vs. diskontinuierliche Strukturierung) gleichermaßen zu berücksichtigen. Die Beurteilung kann für jede Elternperson getrennt vorgenommen werden. Für die verschiedenen Merkmalsbereiche (Achsen und Subachsen) lassen sich die entsprechenden Einzelurteile jeweils zu einer Gesamteinschätzung aggregieren, die man auf der beschriebenen Ordinalskala eintragen kann. Auch das Ausmaß und die Art von (ggf. fehlenden) Übereinstimmungen zwischen den Merkmalsträgern (Mutter, Vater) lassen sich so bestimmen. Damit ergibt sich ein diagnostisches Urteil über die Funktionalität des jeweils untersuchten Realitätsbereiches. Das ermittelte Funktionalitätsniveau wiederum verweist auf mögliche Entwicklungs- und Hilfeziele und auf damit im Zusammenhang stehende Interventionsmöglichkeiten (worauf die wir hier aus Platzgründen nicht weiter eingehen können).

Der differenzierte Beurteilungsvorgang mag auf den ersten Blick abschreckend, weil sehr aufwendig, wirken. Er lässt sich jedoch mit Hilfe des dem Buch von Jacob \& Wahlen (2006) beiliegenden Manuals relativ leicht aneignen. Das Diagnosesystem ist zudem so angelegt, dass der Diagnostiker den im konkreten Einzelfall erforderlichen Aufwand kalkulieren und darüber selbst entscheiden kann - in Abhängigkeit von der benötigten diagnostischen Tiefenschärfe (vom Eindrucksurteil bis zur umfassenden Abklärung). Wir sind überzeugt, dass sich das Klassifikationsund Diagnosesystem - nicht zuletzt unterstützt durch ein Computerprogramm, das sich zurzeit in Entwicklung befindet - gut in die Praxis einfügen wird. Die Einschätzung und Verbesserung der diagnostischen Gütekriterien wird Gegenstand weiterer Untersuchungen sein. 